KYUNGPOOK Math. J. 53(2013), 273-283

http://dx.doi.org/10.5666/KMJ.2013.53.2.273

\title{
Sets of Integer Matrix Pairs Derived from Row Rank Inequal- ities and Their Preservers
}

\author{
SEOK-Zun SonG* \\ Department of Mathematics, Jeju National University, Jeju 690-756, Republic of \\ Korea \\ e-mail: szsong@jejunu.ac.kr \\ YOUNG-BAE JUN \\ Department of Mathematics, Gyeongsang National University, Chinju 660-701, Re- \\ public of Korea \\ e-mail : ybjun@gsnu.ac.kr
}

Abstract. In this paper, we consider the row rank inequalities derived from comparisons of the row ranks of the additions and multiplications of nonnegative integer matrices and construct the sets of nonnegative integer matrix pairs which is occurred at the extreme cases for the row rank inequalities. We characterize the linear operators that preserve these extreme sets of nonnegative integer matrix pairs

\section{Introduction}

The linear preserver problems are one of the most active research subjects in matrix theory during last one hundred years, which concern the characterizations of linear operators on matrix spaces that leave certain functions, subsets, relations, etc., invariant. For survey of these types of problems, we refer to papers in [6]. The specified frame of problems is of interest both for matrices with entries from a field and for matrices with entries from an arbitrary semiring such as Boolean algebra, nonnegative integers, and fuzzy semiring. It is necessary to note that there are several rank functions over a semiring that are analogues of the classical function of the matrix rank over a field. Detailed research and self-contained information about rank functions over semirings can be found in [1] and [5].

There are some results on the inequalities for the rank function of matrices( see [1] - [4]). Beasley and Guterman ([1]) investigated the rank inequalities of matrices over semirings. And they characterized the equality cases for some rank inequalities

\footnotetext{
* Corresponding Author.

Received June 17, 2011; accepted August 23, 2012.

2010 Mathematics Subject Classification: 15A86, 15A03, 15A04.

Key words and phrases: semiring, linear operator, row rank, $(P, Q)$-operator.

This research was supported by the 2013 scientific promotion program funded by Jeju National University.
} 
in [3].

In this paper, we construct the sets of nonnegative integer matrix pairs. These sets are naturally occurred at the extreme cases for the row rank inequalities derived from the addition and multiplication of nonnegative integer matrix pairs. We characterize the linear operators that preserve these extreme sets of nonnegative integer matrix pairs.

A semiring $\mathcal{S}$ consists of a set $\mathcal{S}$ and two binary operations, addition and multiplication, such that:

- $\mathcal{S}$ is an Abelian monoid under addition (identity denoted by 0 );

- $\mathcal{S}$ is an Abelian monoid under multiplication (identity denoted by 1 );

- multiplication is distributive over addition;

- $s 0=0 s=0$ for all $s \in \mathcal{S}$.

Let $z^{+}$be the set of nonnegative integers. Then $z^{+}$becomes a semiring under the usual addition and multiplication. In this thesis we will study the matrices over the semiring $z^{+}$.

A semiring is called antinegative if the zero element is the only element with an additive inverse.

It is straightforward to see that the nonnegative integer semiring $z^{+}$is antinegative.

Let $\mathcal{M}_{m, n}(\mathcal{S})$ denote the set of $m \times n$ matrices with entries from a semiring $\mathcal{S}$. If $m=n$, we use the notation $\mathcal{M}_{n}(\mathcal{S})$ instead of $\mathcal{M}_{n, n}(\mathcal{S})$.

A vector space is usually only defined over fields or division rings, and modules are generalizations of vector spaces defined over rings. We generalize the concept of vector spaces to semiring vector spaces defined over arbitrary semirings.

If $V$ is a nonempty subset of $\mathcal{M}_{m, 1}(\mathcal{S}) \equiv S^{m}$ that is closed under addition and multiplication by scalars, then $V$ is called a semiring vector space over $\mathcal{S}$. The notions of subspace and of spanning sets are the same as if $\mathcal{S}$ were a field.

A subset $W$ of a vector space $V$ is linearly dependent if there exists $\boldsymbol{x} \in W$ such that $\boldsymbol{x}$ is a linear combination of elements in $W \backslash\{\boldsymbol{x}\}$. Otherwise $W$ is linearly independent. Thus an independent set cannot contain a zero vector. As with fields, a basis for a semiring vector space $V$ is a spanning subset of least cardinality. That cardinality is the dimension, $\operatorname{dim}(V)$, of $V$.

The following rank functions are usual in the semiring context.

The matrix $A \in \mathcal{M}_{m, n}(\mathcal{S})$ is said to be of factor rank $k(\operatorname{rank}(A)=k)$ if there exist matrices $B \in \mathcal{M}_{m, k}(\mathcal{S})$ and $C \in \mathcal{M}_{k, n}(\mathcal{S})$ such that $A=B C$ and $k$ is the smallest positive integer for which such factorization exists. By definition, the only matrix with factor rank 0 is the zero matrix, $O$.

The row space of a matrix $A \in \mathcal{M}_{m, n}(\mathcal{S})$ is the semiring vector space that is spanned by its rows. The row rank, $r(A)$, of $A \in \mathcal{M}_{m, n}\left(Z^{+}\right)$is the dimension of its row space. Similarly the column rank, $c(A)$, of $A \in \mathcal{M}_{m, n}\left(\mathcal{Z}^{+}\right)$is the dimension of its column space. 
The matrix $A \in \mathcal{M}_{m, n}(\mathcal{S})$ is said to be of term rank $k(t(A)=k)$ if the least number of lines needed to include all nonzero elements of $A$ is equal to $k$.

The row(column, factor, term) rank of a zero matrix is zero.

Example 1.1. It follows that

$$
1 \leq \operatorname{rank}(A) \leq r(A) \leq n
$$

for all nonzero matrix $A \in \mathcal{M}_{m, n}\left(z^{+}\right)$. These inequalities may be strict: let

$$
A=\left[\begin{array}{ll}
3 & 3 \\
4 & 4
\end{array}\right]=\left[\begin{array}{l}
3 \\
4
\end{array}\right] \times\left[\begin{array}{ll}
1 & 1
\end{array}\right] \in \mathcal{M}_{2,2}\left(Z^{+}\right) .
$$

Then $\operatorname{rank}(A)=1<2=r(A)$ over $z^{+}$.

A line of a matrix $A$ is a row or a column of the matrix $A$.

If $\mathcal{S}$ is a subsemiring of a field then there is a usual rank function $\rho(A)$ for any matrix $A \in \mathcal{M}_{m, n}(\mathcal{S})$. Easy examples show that over semirings these functions are not equal in general. However, the inequalities $r(A) \geq \rho(A)$ and $c(A) \geq \rho(A)$ always hold.

It is well-known that the behavior of the function $\rho$ with respect to matrix addition and multiplication is given by the following inequalities([3]):

- the rank-sum inequalities:

$$
|\rho(A)-\rho(B)| \leq \rho(A+B) \leq \rho(A)+\rho(B),
$$

- Sylvester's laws:

$$
\rho(A)+\rho(B)-n \leq \rho(A B) \leq \min \{\rho(A), \rho(B)\} .
$$

where $A, B$ are conformal matrices with entries from a field.

Arithmetic properties of row rank (or column rank, factor rank) depend on the structure of semiring of entries. It is restricted by the following list of inequalities established in [1]:

For $0 \neq A, B \in \mathcal{M}_{m, n}\left(z^{+}\right)$,

1. $1 \leq r(A+B)$;

2. $r(A+B) \leq m$;

3. $r(A+B) \geq|\rho(A)-\rho(B)|$.

And for $A, B \in \mathcal{M}_{m}\left(Z^{+}\right)$one has that

4. $r(A B) \leq r(A)$;

5. if $\rho(A)+\rho(B)>m$ then $r(A B) \geq \rho(A)+\rho(B)-m$. 
As it was proved in [1] the above inequalities $1 \sim 5$ are sharp and the best possible. The natural question is to characterize the equality cases in the above inequalities. Even over fields this is an open problem, see [2, 3, 4] for more details. The structure of matrix varieties which arise as extremal cases in these inequalities is far from being understood over fields, as well as over semirings. A usual way to generate elements of such a variety is to find a tuple of matrices which belongs to it and to act on this tuple by various linear operators that preserve this variety. The investigation of the corresponding problems over semirings for the factor rank function, term and zero term rank functions was done in $[2,3]$. This paper is a continuation of $[2,3,4]$ and is devoted to study linear operators that preserve extremal cases of row rank inequalities. The complete classification of linear operators that preserve cases of equalities in various matrix inequalities over fields was obtained in $[2,3,4]$. For the details on linear operators preserving matrix invariants one can see $[6]$ and references therein.

\section{Preliminaries}

An operator $T: \mathcal{M}_{m, n}\left(Z^{+}\right) \rightarrow \mathcal{M}_{m, n}\left(Z^{+}\right)$is called linear if $T(\alpha X)=\alpha T(X)$ and $T(X+Y)=T(X)+T(Y)$ for all $X, Y \in \mathcal{M}_{m, n}\left(\mathcal{Z}^{+}\right), \alpha \in \mathcal{S}$.

We say that an operator $T: \mathcal{M}_{m, n}\left(Z^{+}\right) \rightarrow \mathcal{M}_{m, n}\left(Z^{+}\right)$preserves a set $\mathcal{P}$ if $X \in \mathcal{P}$ implies that $T(X) \in \mathcal{P}$, or, if $\mathcal{P}$ is a set of ordered pairs [triples], that $(X, Y) \in \mathcal{P}$ $[(X, Y, Z) \in \mathcal{P}]$ implies $(T(X), T(Y)) \in \mathcal{P}[(T(X), T(Y), T(Z)) \in \mathcal{P}]$.

The matrix $X \circ Y$ denotes the Hadamard or Schur product, i.e., the $(i, j)$ entry of $X \circ Y$ is $x_{i, j} y_{i, j}$.

An operator $T: \mathcal{M}_{m, n}\left(Z^{+}\right) \rightarrow \mathcal{M}_{m, n}\left(Z^{+}\right)$is called a $(P, Q, B)$-operator if there exist permutation matrices $P \in \mathcal{M}_{m}\left(z^{+}\right)$and $Q \in \mathcal{M}_{n}\left(z^{+}\right)$, and a matrix $B=$ $\left[b_{i, j}\right] \in \mathcal{M}_{m, n}\left(z^{+}\right), b_{i, j}$ are nonzero elements from $z^{+}$for all $i, j, 1 \leq i \leq m$, $1 \leq j \leq n$, such that $T(X)=P(X \circ B) Q$ for all $X \in \mathcal{M}_{m, n}\left(z^{+}\right)$or when $m=n$ $T(X)=P(X \circ B)^{t} Q$ for all $X \in \mathcal{M}_{n}\left(Z^{+}\right)$where $X^{t}$ denotes the transpose of $X$. An operator $T$ is called a nontransposing $(P, Q, B)$-operator if there exist permutation matrices $P \in \mathcal{M}_{n}\left(z^{+}\right)$and $Q \in \mathcal{M}_{n}\left(z^{+}\right)$, and a matrix $B=\left[b_{i, j}\right] \in \mathcal{M}_{m, n}\left(z^{+}\right)$, $b_{i, j}$ are nonzero elements from $Z^{+}$for all $i, j, 1 \leq i \leq m, 1 \leq j \leq n$, such that $T(X)=P(X \circ B) Q$ for all $X \in \mathcal{M}_{m, n}\left(z^{+}\right)$. For the case of $B=J,(P, Q, B)$ operator is called $(P, Q)$-operator.

We say that the matrix $A$ dominates the matrix $B$ if and only if $b_{i, j} \neq 0$ implies that $a_{i, j} \neq 0$, and we write $A \geq B$ or $B \leq A$ in this case.

If $A$ and $B$ are matrices and $A \geq B$ we let $A \backslash B$ denote the matrix $C$ where

$$
c_{i, j}=\left\{\begin{array}{rl}
0 & \text { if } b_{i, j} \neq 0 \\
a_{i, j} & \text { otherwise }
\end{array} .\right.
$$

The matrix $I_{n}$ is the $n \times n$ identity matrix, $J_{m, n}$ is the $m \times n$ matrix of all ones, $O_{m, n}$ is the $m \times n$ zero matrix. We omit the subscripts when the order is obvious from the context and we write $I, J$, and $O$, respectively. The matrix $E_{i, j}$, called a cell, denotes the matrix with 1 in $(i, j)$ position and zero elsewhere. A weighted 
cell is any nonzero scalar multiple of a cell, i.e., $\alpha E_{i, j}$ is a weighted cell for any $0 \neq \alpha \in z^{+}$. Let $R_{i}$ denote the matrix whose $i^{\text {th }}$ row is all ones and all other rows are zero, and $C_{j}$ denote the matrix whose $j^{\text {th }}$ column is all ones and all other columns are zero. We let $|A|$ denote the number of nonzero entries in the matrix $A$. We denote by $A\left[i_{1}, \ldots, i_{k} \mid j_{1}, \ldots, j_{l}\right]$ the $k \times l$-submatrix of $A$ which lies in the intersection of the $i_{1}, \ldots, i_{k}$ rows and $j_{1}, \ldots, j_{l}$ columns.

We obtain some basic results on the linear operators on $\mathcal{M}_{m, n}\left(\mathcal{Z}^{+}\right)$for later use.

Theorem 2.1. Let $T: \mathcal{M}_{m, n}\left(\mathcal{Z}^{+}\right) \rightarrow \mathcal{M}_{m, n}\left(\mathcal{Z}^{+}\right)$be a linear operator. Then the following are equivalent:

(1) $T$ is bijective;

(2) $T$ is surjective;

(3) There exists a permutation $\sigma$ on $\{(i, j) \mid i=1,2, \ldots, m ; j=1,2, \ldots, n\}$ such that $T\left(E_{i, j}\right)=E_{\sigma(i, j)}$.

Proof. It is trivial that (1) implies (2) and (3) implies (1).

We now show that (2) implies (3).

We assume that $T$ is surjective. Then, for any pair $(i, j)$, there exists some $X$ such that $T(X)=E_{i, j}$. Clearly $X \neq O$ by the linearity of $T$. Thus there is a pair of indices $(r, s)$ such that $X=x_{r, s} E_{r, s}+X^{\prime}$ where $(r, s)$ entry of $X^{\prime}$ is zero and the following two conditions are satisfied: $x_{r, s} \neq 0$ and $T\left(E_{r, s}\right) \neq O$. Indeed, if in the contrary for all pairs $(r, s)$ either $x_{r, s}=0$ or $T\left(E_{r, s}\right)=O$ then $T(X)=0$ which contradicts with the assumption $T(X)=E_{i, j}$. Since $z^{+}$is antinegative without zero divisors it follows that

$$
T\left(x_{r, s} E_{r, s}\right) \leq T\left(x_{r, s} E_{r, s}\right)+T\left(X \backslash\left(x_{r, s} E_{r, s}\right)\right)=T(X)=E_{i, j} .
$$

Hence, $x_{r, s} T\left(E_{r, s}\right)=T\left(x_{r, s} E_{r, s}\right) \leq E_{i, j}$ and $T\left(E_{r, s}\right) \neq O$ by the above. Therefore, $T\left(E_{r, s}\right) \leq E_{i, j}$. Indeed, if on the contrary, $T\left(E_{r, s}\right)$ is a sum of certain multiples of cells then so is $x_{r, s} T\left(E_{r, s}\right)$, since $\mathcal{Z}^{+}$is antinegative and without zero divisors.

Let $\Xi_{i, j}=\left\{E_{r, s} \mid T\left(E_{r, s}\right) \leq E_{i, j}\right\}$. By the above $\Xi_{i, j} \neq \emptyset$ for all $(i, j)$. By its definition $\Xi_{i, j} \cap \Xi_{u, v}=\emptyset$ whenever $(i, j) \neq(u, v)$. That is $\left\{\Xi_{i, j}\right\}$ is a set of $m n$ nonempty cells which partition the set of cells. By the pigeonhole principle, we must have that $\left|\Xi_{i, j}\right|=1$ for all $(i, j)$. Necessarily, for each pair $(r, s)$ there is a unique pair $(i, j)$ such that $T\left(E_{r, s}\right)=b_{r, s} E_{i, j}$. That is there is some permutation $\sigma$ on $\{(i, j) \mid i=1,2, \cdots, m ; j=1,2, \cdots, n\}$ such that for some scalars $b_{i, j}, T\left(E_{i, j}\right)=$ $b_{i, j} E_{\sigma(i, j)}$. We now only need to show that the $b_{i, j}=1$. Since $T$ is surjective and $T\left(E_{r, s}\right) \not \leq E_{\sigma(i, j)}$ for $(r, s) \neq(i, j)$, there is some $\alpha$ such that $T\left(\alpha E_{i, j}\right)=E_{\sigma(i, j)}$. But then, since $T$ is linear, $E_{\sigma(i, j)}=T\left(\alpha E_{i, j}\right)=\alpha T\left(E_{i, j}\right)=\alpha b_{i, j} E_{\sigma(i, j)}$. That is, $\alpha b_{i, j}=1$, or $b_{i, j}=1$ in $z^{+}$.

Lemma 2.2. Let $T: \mathcal{M}_{m, n}\left(\mathcal{Z}^{+}\right) \rightarrow \mathcal{M}_{m, n}\left(\mathcal{Z}^{+}\right)$be an operator which maps lines to lines and is defined by $T\left(E_{i, j}\right)=E_{\sigma(i, j)}$, where $\sigma$ is a permutation on the set $\{(i, j) \mid i=1,2, \ldots, m ; j=1,2, \ldots, n\}$. Then $T$ is a $(P, Q)$-operator. 
Proof. Since no combination of $a$ rows and $b$ columns can dominate $J$ where $a+b=$ $m$ unless $b=0$ (or if $m=n$, if $a=0$ ) we have that either the image of each row is a row and the image of each column is a column, or $m=n$ and the image of each row is a column and the image of each column is a row. Thus, there are permutation matrices $P$ and $Q$ such that $T\left(R_{i}\right) \leq P R_{i} Q$ and $T\left(C_{j}\right) \leq P C_{j} Q$ or, if $m=n$, $T\left(R_{i}\right) \leq P\left(R_{i}\right)^{t} Q$ and $T\left(C_{j}\right) \leq P\left(C_{j}\right)^{t} Q$. Since each cell lies in the intersection of a row and a column and $T$ maps nonzero cells to nonzero cells, it follows that $T\left(E_{i, j}\right)=P E_{i, j} Q$, or, if $m=n, T\left(E_{i, j}\right)=P E_{j, i} Q=P\left(E_{i, j}\right)^{t} Q$.

Remark 2.3. One can easily check that if $m=1$ or $n=1$ then all operators under consideration are $(P, Q)$-operators, if $m=n=1$ then all operators under consideration are $\left(P, P^{t}\right)$-operators.

Henceforth we will always assume that $m, n \geq 2$.

Now let us construct the set of matrix pairs that arise as extremal cases in the inequalities $1 \sim 5$ in section 1 on matrices over $z^{+}$:

$$
\begin{gathered}
\mathcal{R}_{A m}\left(z^{+}\right)=\left\{(X, Y) \in \mathcal{M}_{m, n}\left(Z^{+}\right)^{2} \mid r(X+Y)=m\right\} ; \\
\mathcal{R}_{A 1}\left(Z^{+}\right)=\left\{(X, Y) \in \mathcal{M}_{m, n}\left(z^{+}\right)^{2} \mid r(X+Y)=1\right\} ; \\
\mathcal{R}_{A \rho}\left(Z^{+}\right)=\left\{(X, Y) \in \mathcal{M}_{m, n}\left(z^{+}\right)^{2}|r(X+Y)=| \rho(X)-\rho(Y) \mid\right\} ; \\
\mathcal{R}_{M l}\left(z^{+}\right)=\left\{(X, Y) \in \mathcal{M}_{m}\left(z^{+}\right)^{2} \mid r(X Y)=r(X)\right\} ; \\
\mathcal{R}_{M \rho}\left(Z^{+}\right)=\left\{(X, Y) \in \mathcal{M}_{m}\left(z^{+}\right)^{2} \mid r(X Y)=\rho(X)+\rho(Y)-m\right\} .
\end{gathered}
$$

\section{Linear operators preserving extreme set of integer matrix pairs}

In this section, we obtain the characterizations of the linear operators that preserve the extreme sets $\mathcal{R}_{* *}\left(Z^{+}\right)$defined in section 2 .

\subsection{Linear operators that preserve $\mathcal{R}_{A m}\left(Z^{+}\right)$}

Lemma 3.1. If $T: \mathcal{M}_{m, n}\left(Z^{+}\right) \rightarrow \mathcal{M}_{m, n}\left(Z^{+}\right)$is a surjective linear operator which preserves $\mathcal{R}_{A m}\left(\mathcal{Z}^{+}\right)$, then $T$ maps lines to lines.

Proof. Suppose that $T^{-1}$ does not map lines to lines. Then, there are two non collinear cells which are mapped to a line. There are two cases, they are mapped to two cells in a row or two cells in a column by Theorem 2.1.

If two non-collinear cells are mapped to two cells in a row, we may assume without loss of generality that $T\left(E_{1,1}+E_{2,2}\right)=E_{1,1}+E_{1,2}$. If $m \leq n$ it suffices to consider $A=E_{1,1}+E_{2,2}+\ldots+E_{m, m}$. In this case, $T(A)$ has row rank at most $m-1$, i.e., $(0, A) \in \mathcal{R}_{A m}\left(z^{+}\right),(0, T(A)) \notin \mathcal{R}_{A m}\left(z^{+}\right)$, a contradiction. 
Let us consider the case $n<m$. Then we choose a matrix $A^{\prime} \in \mathcal{M}_{m-2, n-2}\left(Z^{+}\right)$ such that

$$
A^{\prime}=\left[\begin{array}{cccc}
m & 0 & \cdots & 0 \\
m+1 & 0 & \cdots & 0 \\
\cdots \cdots & & & \\
2 m-3 & 0 & \cdots & 0
\end{array}\right] \in \mathcal{M}_{m-2, n-2}\left(z^{+}\right) .
$$

Then $r\left(A^{\prime}\right)=m-2$. Let $A=O_{2} \oplus A^{\prime} \in \mathcal{M}_{m, n}\left(Z^{+}\right)$. Thus $r(A)=r\left(A^{\prime}\right)=m-2$. Hence $\left(E_{1,1}+E_{2,2}, A\right) \in \mathcal{R}_{A m}\left(z^{+}\right)$. Since $T$ preserves $\mathcal{R}_{A m}\left(z^{+}\right)$, it follows that $\left(E_{1,1}+E_{1,2}, T(A)\right) \in \mathcal{R}_{A m}\left(Z^{+}\right)$, i.e., $r\left(E_{1,1}+E_{1,2}+T(A)\right)=m$. Therefore $r(T(A)[3, \ldots, m ; 1, \ldots, n]) \geq m-2$. Since the row rank of any matrix cannot exceed the number of rows, $r(T(A)[3, \ldots, m ; 1, \ldots, n])=m-2$. Further, $|T(A)[3, \ldots, m ; 1, \ldots, n]|<|A|=\left|A^{\prime}\right|=m-2$ since $T$ transforms weighted cells to weighted cells and at least one cell has to be mapped into the $2^{\text {nd }}$ row. Thus we can have an $(m-2) \times n$ submatrix of $E_{1,1}+E_{1,2}+T(A)$ whose row rank is $m-2$ and the number of whose nonzero entries are less than $m-2$. This is a contradiction.

If two non-collinear cells are mapped to two cells in a column, we may assume without loss of generality that $T\left(E_{1,1}+E_{2,2}\right)=E_{1,1}+E_{2,1}$. In this case, by considering the matrices $E_{1,1}+E_{2,2}$ and $A$ chosen above, the result follows.

Thus, $T$ maps lines to lines.

Theorem 3.2. Let $T: \mathcal{M}_{m, n}\left(\mathcal{Z}^{+}\right) \rightarrow \mathcal{M}_{m, n}\left(\mathcal{Z}^{+}\right)$be a surjective linear operator. Then $T$ preserves $\mathcal{R}_{A m}\left(Z^{+}\right)$if and only if $T$ is a nontransposing $(P, Q)$-operator.

Proof. It is easily checked that all nontransposing $(P, Q)$-operators preserve $\mathcal{R}_{A m}\left(z^{+}\right)$.

Suppose that $T$ preserves $\mathcal{R}_{A m}\left(Z^{+}\right)$. By Lemma 3.1 we have that $T$ maps lines to lines and by applying Theorem 2.1 to Lemma 2.2 we have that $T$ is a $(P, Q)$ operator. Since all nontransposing $(P, Q)$-operators preserve $\mathcal{R}_{A m}\left(Z^{+}\right)$it only remains to show that if $m=n$ then the transposition does not preserve $\mathcal{R}_{A m}\left(z^{+}\right)$. Let $A=\left[\begin{array}{ll}3 & 3 \\ 4 & 4\end{array}\right] \oplus I_{m-2} \in \mathcal{M}_{m}\left(z^{+}\right)$. Then by Example 1.1 we have that $r(A)=m$ and $r\left(A^{t}\right)=m-1$, so that $(A, O) \in \mathcal{R}_{A m}\left(Z^{+}\right)$while $\left(A^{t}, O\right) \notin \mathcal{R}_{A m}\left(Z^{+}\right)$. Thus $T$ is a nontransposing $(P, Q)$-operator.

\subsection{Linear operators that preserve $\mathcal{R}_{A 1}\left(z^{+}\right)$}

Lemma 3.3. If $T: \mathcal{M}_{m, n}\left(\mathcal{Z}^{+}\right) \rightarrow \mathcal{M}_{m, n}\left(Z^{+}\right)$is a surjective linear operator which preserves $\mathcal{R}_{A 1}\left(Z^{+}\right)$, then $T$ maps lines to lines.

Proof. Suppose that $T$ does not map lines to lines. Then, without loss of generality, we may assume that either $T\left(E_{1,1}+E_{1,2}\right)=E_{1,1}+E_{2,2}$ or $T\left(E_{1,1}+E_{2,1}\right)=$ $E_{1,1}+E_{2,2}$ by Theorem 2.1. In either case, let $Y=O$ and $X$ be either $E_{1,1}+E_{1,2}$ or $E_{1,1}+E_{2,1}$, so that $(X, Y) \in \mathcal{R}_{A 1}\left(Z^{+}\right)$while $(T(X), T(Y)) \notin \mathcal{R}_{A 1}\left(Z^{+}\right)$, a contradiction. Thus $T$ maps lines to lines.

Theorem 3.4. Let $T: \mathcal{M}_{m, n}\left(\mathcal{Z}^{+}\right) \rightarrow \mathcal{M}_{m, n}\left(Z^{+}\right)$be a surjective linear operator. 
Then $T$ preserves $\mathcal{R}_{A 1}\left(Z^{+}\right)$if and only if $T$ is a nontransposing $(P, Q)$-operator.

Proof. It is easily checked that all nontransposing $(P, Q)$-operators preserve $\mathcal{R}_{A 1}\left(z^{+}\right)$.

Suppose that $T$ preserves $\mathcal{R}_{A 1}\left(z^{+}\right)$. By applying Lemma 3.3 and Theorem 2.1 to Lemma 2.2 we have that if $T$ preserves $\mathcal{R}_{A 1}\left(Z^{+}\right)$then $T$ is a $(P, Q)$-operator. Since all nontransposing $(P, Q)$-operators preserve $\mathcal{R}_{A 1}\left(Z^{+}\right)$it only remains to show that if $m=n$ then the transposition does not preserve $\mathcal{R}_{A 1}\left(Z^{+}\right)$. Let $X=\left[\begin{array}{cc}3 & 4\end{array}\right] \bigoplus O_{m-1, m-2} \in \mathcal{M}_{m}\left(Z^{+}\right)$and $Y=O \in \mathcal{M}_{m}\left(Z^{+}\right),(X, Y) \in \mathcal{R}_{A 1}\left(Z^{+}\right)$ but $\left(X^{t}, Y^{t}\right) \notin \mathcal{R}_{A 1}\left(Z^{+}\right)$. So, transposition operator does not preserve the set $\mathcal{R}_{A 1}\left(Z^{+}\right)$. Thus $T$ is a nontransposing $(P, Q)$-operator.

\subsection{Linear operators that preserve $\mathcal{R}_{A \rho}\left(z^{+}\right)$}

Lemma 3.5. If $T: \mathcal{M}_{m, n}\left(Z^{+}\right) \rightarrow \mathcal{M}_{m, n}\left(Z^{+}\right)$is a surjective linear operator which preserves $\mathcal{R}_{A \rho}\left(\mathcal{Z}^{+}\right), \min \{m, n\} \geq 3$, then $T$ maps lines to lines.

Proof. The sum of three distinct weighted cells has row rank at most 3 . Thus $T\left(E_{1,1}+E_{1,2}+E_{2,1}\right)$ is either a sum of 3 collinear cells, and hence has row rank 1 , or is contained in two lines, and hence has real rank 2 , or is the sum of three cells of row rank 3 and hence of real rank 3. Now, for $X=E_{1,1}+E_{1,2}+E_{2,1}$ and $Y=E_{2,2}$, we have that $(X, Y) \in \mathcal{R}_{A R}\left(Z^{+}\right)$, and the image of $Y$ is a single cell, and hence $\rho(T(Y))=1$. Now, if $\rho(T(X))=3$, then $T(X+Y)$ must have row rank 3 or 4 , and hence $(T(X), T(Y)) \notin \mathcal{R}_{A R}\left(Z^{+}\right)$, a contradiction. If $\rho(T(X))=1$, clearly $(T(X), T(Y)) \notin \mathcal{R}_{A R}\left(\mathcal{Z}^{+}\right)$since $T(X+Y) \neq O$. Thus $\rho(T(X))=2$, and $r(T(X+Y))=1$. However it is straightforward to see that the sum of four cells has the column rank 1 if and only if they lie either in a line or in the intersection of two rows and two columns. The matrix $T(X+Y)$ is a sum of four cells. These cells do not lie in a line since $\rho(T(X))=2$. Thus $T(X+Y)$ must be the sum of four cells which lie in the intersection of two rows and two columns. Similarly, for any $i, j, k, l, T\left(E_{i, j}+E_{i, k}+E_{l, j}+E_{l, k}\right)$ must lie in the intersection of two rows and two columns. It follows that any two rows must be mapped into two lines. Since $T$ is bijective, if some pair of two rows is mapped into two rows (resp. columns), any pair of two rows is mapped into two rows (resp. columns). Similarly, if some pair of two columns is mapped into two rows (resp. columns), any pair of two columns is mapped into two rows (resp. columns).

Now, the image of three rows is contained in three lines, two of which are the image of two rows, thus, every row is mapped into a line. Similarly for columns. Thus, $T$ maps lines to lines.

Theorem 3.6. Let $T: \mathcal{M}_{m, n}\left(\mathcal{Z}^{+}\right) \rightarrow \mathcal{M}_{m, n}\left(\mathcal{Z}^{+}\right)$be a surjective linear operator. Then $T$ preserves $\mathcal{R}_{A \rho}\left(Z^{+}\right)$if and only if $T$ is a nontransposing $(P, Q)$-operator.

Proof. It is easily checked that all nontransposing $(P, Q)$-operators preserve $\mathcal{R}_{A \rho}\left(z^{+}\right)$.

By applying Lemma 3.5 and Theorem 2.1 to Lemma 2.2 we have that if $T$ preserves $\mathcal{R}_{A \rho}\left(Z^{+}\right)$then $T$ is a $(P, Q)$-operator. Since all nontransposing $(P, Q)$ - 
operators preserve $\mathcal{R}_{A \rho}\left(z^{+}\right)$it only remains to show that in the case $m=n$ the operator $X \rightarrow X^{t}$ does not preserve $\mathcal{R}_{A \rho}\left(Z^{+}\right)$. Let $X=\left[\begin{array}{ll}3 & 4 \\ 3 & 4\end{array}\right] \oplus O_{m-2, m-2} \in$ $\mathcal{M}_{m}\left(z^{+}\right)$and $Y=O_{m, m} \in \mathcal{M}_{m}\left(z^{+}\right)$. Then $r(X+Y)=1=\rho(X)-\rho(Y)$ and hence $(X, Y) \in \mathcal{R}_{A \rho}\left(Z^{+}\right)$while $r\left(X^{t}+Y^{t}\right)=2 \neq 1=\rho(X)-\rho(Y)$ so that $\left(X^{t}, Y^{t}\right) \notin$ $\mathcal{R}_{A \rho}\left(Z^{+}\right)$. So, transposition operator does not preserve the set $\mathcal{R}_{A \rho}\left(Z^{+}\right)$. Thus $T$ is a nontransposing $(P, Q)$-operator.

\subsection{Linear operators that preserve $\mathcal{R}_{M l}\left(z^{+}\right)$}

Lemma 3.7. If $T: \mathcal{M}_{m}\left(Z^{+}\right) \rightarrow \mathcal{M}_{m}\left(Z^{+}\right)$is a surjective linear operator which preserves $\mathcal{R}_{M l}\left(Z^{+}\right)$, then $T$ maps lines to lines.

Proof. Suppose that $T^{-1}$ does not map rows to lines, say, without loss of generality, that $T^{-1}\left(E_{1,1}+E_{1,2}\right) \geq E_{1,1}+E_{2,2}$. Then $T(I)$ has nonzero entries in at most $m-1$ rows. Suppose $T(I)$ has all zero entries in row $j$. Then for $X=T^{-1}\left(E_{1, j}\right)$ and $Y=I$, we have $X Y=X$ and hence $(X, Y) \in \mathcal{R}_{M l}\left(z^{+}\right)$however, $T(X) T(Y)=O$ and hence $(T(X), T(Y)) \notin \mathcal{R}_{M l}\left(Z^{+}\right)$. This contradicts the fact that $T$ preserves $\mathcal{R}_{M l}\left(z^{+}\right)$.

Suppose that $T^{-1}$ does not map columns to lines. Say, without loss of generality, that $T^{-1}\left(E_{1,1}+E_{2,1}\right) \geq E_{1,1}+E_{2,2}$. That is $T\left(E_{1,1}+E_{2,2}\right)=E_{1,1}+E_{2,1}$. Then for $B=I, T(B)$ has row rank at most $m-1$ since either the first two rows of $T(B)$ are equal or at least one of the rows from the $3^{\text {rd }}$ through the $m^{\text {th }}$ is zero. Let $A=T^{-1}(I)$, then we have that $A B=A$ and hence $(A, B) \in \mathcal{R}_{M l}\left(Z^{+}\right)$, while $r(T(A) T(B))=r(T(B)) \leq m-1<r(I)=r(T(A))$ so that $(T(A), T(B)) \notin$ $\mathcal{R}_{M l}\left(Z^{+}\right)$, a contradiction.

Thus $T^{-1}$ and hence $T$ map lines to lines.

Theorem 3.8. Let $T: \mathcal{M}_{m}\left(z^{+}\right) \rightarrow \mathcal{M}_{m}\left(z^{+}\right)$be a surjective linear operator. Then $T$ preserves $\mathcal{R}_{M l}\left(Z^{+}\right)$if and only if $T$ is a nontransposing $\left(P, P^{t}\right)$-operator.

Proof. It is easily checked that all nontransposing $(P, Q)$-operators preserve $\mathcal{R}_{M l}\left(Z^{+}\right)$.

By applying Lemma 3.7 and Theorem 2.1 to Lemma 2.2 we have that if $T$ preserves $\mathcal{R}_{M l}\left(Z^{+}\right)$then $T$ is a $(P, Q)$-operator.

To prove that $Q=P^{t}$, assume on the contrary that $Q P \neq I$. Suppose that $T_{1}(X)=(Q P) X$ transforms the $r^{\text {th }}$ row into the $t^{\text {th }}$ row for some $r \neq t$. We consider the matrix $X=E_{t, t}, \quad Y=I \backslash E_{r, r}$. Then $X Y=E_{t, t}=X$, which implies $(X, Y) \in \mathcal{R}_{M l}\left(Z^{+}\right)$, but $T(X) T(Y)=P X Q P Y Q=P 0 Q=0$, since all the entries of the $t^{\text {th }}$ row of $Q P Y$ are zero. Thus $(T(X), T(Y)) \notin \mathcal{R}_{M l}\left(Z^{+}\right)$. Therefore $T$ does not preserve the set $\mathcal{R}_{M l}\left(Z^{+}\right)$, a contradiction. Thus $Q=P^{t}$ and $T$ is a $\left(P, P^{t}\right)$ operator.

Since all nontransposing $\left(P, P^{t}\right)$-operators preserve $\mathcal{R}_{M l}\left(Z^{+}\right)$it only remains to show that the operator $X \rightarrow X^{t}$ does not preserve $\mathcal{R}_{M l}\left(z^{+}\right)$, since row and column permutations preserve $\mathcal{R}_{M l}\left(\mathcal{Z}^{+}\right)$. Let $X=\left[\begin{array}{ll}1 & 1 \\ 1 & 2\end{array}\right] \oplus I_{m-2}, Y=$ 
$\left[\begin{array}{ll}1 & 0 \\ 1 & 0\end{array}\right] \bigoplus I_{m-2}$. Then $r(X Y)=r\left(\left[\begin{array}{ll}2 & 0 \\ 3 & 0\end{array}\right] \bigoplus I_{m-2}\right)=m=r(X)$ so that $(X, Y) \in \mathcal{R}_{M l}\left(Z^{+}\right)$while $r\left(X^{t} Y^{t}\right)=r\left(\left[\begin{array}{ll}1 & 1 \\ 1 & 1\end{array}\right] \oplus I_{m-2}\right)=m-1 \neq m=r\left(X^{t}\right)$ so that $\left(X^{t}, Y^{t}\right) \notin \mathcal{R}_{M l}\left(Z^{+}\right)$. So, transposition operator does not preserve the set $\mathcal{R}_{M l}\left(Z^{+}\right)$. Thus $T$ is a nontransposing $\left(P, P^{t}\right)$-operator.

\subsection{Linear operators that preserve $\mathcal{R}_{M \rho}\left(z^{+}\right)$}

Lemma 3.9. If $T: \mathcal{M}_{m}\left(Z^{+}\right) \rightarrow \mathcal{M}_{m}\left(Z^{+}\right)$is a surjective linear operator which preserves $\mathcal{R}_{M \rho}\left(\mathcal{Z}^{+}\right)$, then $T$ maps lines to lines.

Proof. If $T$ does not preserve lines, then there exist indices $i, j, k, l, i \neq k, j \neq l$ such that nonzero entries of $T\left(E_{i, j}\right)$ and $T\left(E_{k, l}\right)$ lie in a line. Let $P^{\prime} \in \mathcal{M}_{n}\left(z^{+}\right)$ be a matrix such that $P=P^{\prime}+E_{i, j}+E_{k, l}$ is a permutational matrix. Then $(P, O) \in \mathcal{R}_{M \rho}\left(Z^{+}\right)$. However, $r(T(X)) \leq n-1$, and hence $\rho(T(X)) \leq n-1$. Thus $(T(X), O) \notin \mathcal{R}_{M \rho}\left(Z^{+}\right)$, a contradiction. Thus $T$ maps lines to lines.

Theorem 3.10. Let $T: \mathcal{M}_{m}\left(Z^{+}\right) \rightarrow \mathcal{M}_{m}\left(Z^{+}\right)$be a surjective linear operator. Then $T$ preserves $\mathcal{R}_{M \rho}\left(Z^{+}\right)$if and only if $T$ is a nontransposing $\left(P, P^{t}\right)$-operator.

Proof. It is straightforward that operators under consideration preserve the set $\mathcal{R}_{M \rho}\left(Z^{+}\right)$.

By applying Lemma 3.9 and Theorem 2.1 to Lemma 2.2 we have that if $T$ preserves $\mathcal{R}_{M \rho}\left(Z^{+}\right)$then $T$ is a $(P, Q)$-operator.

Let $X=\left[\begin{array}{ll}0 & 1 \\ 0 & 0\end{array}\right] \oplus I_{n-2}$, and $Y=\left[\begin{array}{ll}1 & 0 \\ 0 & 0\end{array}\right] \oplus I_{n-2}$. Then $X Y=$ $\left[\begin{array}{ll}0 & 0 \\ 0 & 0\end{array}\right] \oplus I_{n-2}$, and hence $(X, Y) \in \mathcal{R}_{M \rho}\left(Z^{+}\right)$. But $X^{t} Y^{t}=\left[\begin{array}{ll}0 & 0 \\ 1 & 0\end{array}\right] \oplus I_{n-2}$, and hence $\left(X^{t}, Y^{t}\right) \notin \mathcal{R}_{M \rho}\left(Z^{+}\right)$. This proves that $T$ is a non-transposing $(P, Q)$ operator.

Let us check that $Q=P^{t}$. Assume in the contrary that $Q P \neq I$. Let $Q P$ transforms $r^{\text {th }}$ row to $t^{\text {th }}$ with $r \neq t$. We consider the matrix $X=I \backslash E_{r, r}$, $Y=E_{r, r}$. Thus $X Y=0$ and hence $(X, Y) \in \mathcal{R}_{M \rho}\left(Z^{+}\right)$. But $T(X) T(Y)=$ $\left.P X Q P Y Q=P\left(I \backslash E_{r, r}\right) E_{t, r}\right) Q \neq 0$ and hence $(T(X), T(Y)) \notin \mathcal{R}_{M \rho}\left(\mathcal{Z}^{+}\right)$. This contradiction proves that $Q=P^{t}$.

\subsection{Examples of non-surjective linear operators that preserve $\mathcal{R}_{* *}\left(z^{+}\right)$}

Let us see that there exist non invertible linear preservers of the sets $\mathcal{R}_{* *}\left(z^{+}\right)$.

\section{Example 3.11.}

1. Let $m \leq n$, and $T_{m}: \mathcal{M}_{m, n}\left(\mathcal{Z}^{+}\right) \rightarrow \mathcal{M}_{m, n}\left(\mathcal{Z}^{+}\right)$be a linear operator which is defined on the basis by $T_{m}\left(E_{i, j}\right)=E_{i, i}$. Then $T_{m}$ is not surjective but it preserves $\mathcal{R}_{A m}\left(Z^{+}\right)$.

2. Let $T_{1}: \mathcal{M}_{m, n}\left(\mathcal{Z}^{+}\right) \rightarrow \mathcal{M}_{m, n}\left(Z^{+}\right)$be a linear operator which is defined on the 
basis by $T_{1}\left(E_{i, j}\right)=E_{1,1}$. Then $T_{1}$ is not surjective but it preserves $\mathcal{R}_{A 1}\left(z^{+}\right)$.

3. Let $T_{\rho}: \mathcal{M}_{m}\left(\mathcal{Z}^{+}\right) \rightarrow \mathcal{M}_{m}\left(\mathcal{Z}^{+}\right)$be a linear operator which is defined by $T_{\rho}(X)=\left(\sum_{i, j=1}^{m} x_{i, j}\right) I_{m}$. Then $T_{\rho}$ is not surjective but it preserves $\mathcal{R}_{M l}\left(Z^{+}\right)$ and $\mathcal{R}_{M \rho}\left(z^{+}\right)$.

Proof.

1. By its definition $T_{m}$ is not surjective. To see that $T_{m}$ preserves $\mathcal{R}_{A m}\left(z^{+}\right)$, choose any $A, B \in \mathcal{M}_{m, n}\left(Z^{+}\right)$with $r(A+B)=m$. Then there is no zero row in $A+B$, and hence $T_{m}(A+B)$ also has no zero rows and the rows are all linearly independent. Thus $r\left(T_{m}(A+B)\right)=m$. Hence, $T_{m}$ preserves $\mathcal{R}_{A m}\left(Z^{+}\right)$.

2. It is trivial that $T_{1}$ is not surjective but preserves $\mathcal{R}_{A 1}\left(Z^{+}\right)$.

3. It is trivial that $T_{\rho}$ is not surjective. If $r(A B)=r(A)$ for some $A, B \in$ $\mathcal{M}_{m}\left(Z^{+}\right)$then $r(T(A) T(B))=r\left(\left(\sum_{i, j=1}^{m} a_{i, j}\right)\left(\sum_{i, j=1}^{m} b_{i, j}\right) I_{m}\right)=m=r(T(A))$ and $r(T(A) T(B))=r\left(\left(\sum_{i, j=1}^{m} a_{i, j}\right)\left(\sum_{i, j=1}^{m} b_{i, j}\right) I_{m}\right)=\rho(T(A))+\rho(T(B))-m$. Thus $T_{\rho}$ preserves $\mathcal{R}_{M l}\left(z^{+}\right)$and $\mathcal{R}_{M \rho}\left(z^{+}\right)$.

\section{References}

[1] L. B. Beasley, A. E. Guterman, Rank inequalities over semirings, Journal of the Korean Math. Soc., 42(2)(2005), 223-241.

[2] L. B. Beasley, A. E. Guterman, Linear preservers of extremes of rank inequalities over semirings, Factor rank, Journal of Mathematical Sciences (New York), 131(2005), 5919-5938.

[3] L. B. Beasley, A. E. Guterman, C. L. Neal, Linear preservers for Sylvester and Frobenius bounds on matrix rank, Rocky Mountains J. of Mathematics, 36(1)(2006), 67-80.

[4] L. B. Beasley, S.-G. Lee, S.-Z. Song, Linear operators that preserve pairs of matrices which satisfy extreme rank properties, Linear Algebra Appl., 350(2002), 263-272.

[5] L. B. Beasley, S. Z. Song, K. T. Kang and B. K. Sarma, Column ranks and their preservers over nonnegative real matrices, Linear Algebra Appl. 399(2005), 3-16.

[6] P. Pierce and others, A Survey of Linear Preserver Problems, Linear and Multilinear Algebra, 33(1992), 1-119. 